\title{
Research on Detection Method of AC/DC Charging Interface Consistency of Electric Vehicles
}

\author{
Kang $\mathrm{Li}^{1,2, a}$, Chang $\mathrm{Liu}^{1,2}$, Lu Xia ${ }^{1,2}$, Bin $\mathrm{Li}^{1,2}$, Yanmin Wang ${ }^{1,2}$, Jing Zhang ${ }^{1,2}$ \\ ${ }^{1}$ China Electric Power Research Institute, Beijing, 100192, China \\ ${ }^{2}$ Beijing Electric Vehicle Charging Battery Swapping Engineering and Technology Research Center, \\ Beijing, 100192, China \\ aemail: saberaward@163.com
}

Keywords: detection system of electric vehicles; charging interface consistency; charging interface compatibility; charging interface

\begin{abstract}
However, in terms of the interchange and versatility of charging interface of electric vehicles, the research on consistency detection technology is not enough, which leads to poor compatibility of the interface of charging device of electric vehicles, thus the use rate of equipment and user satisfaction are reduced, and the popularization and application of electric vehicles is impeded. The detection method put forward in this paper realizes detection on charging interface, charge control and compatibility of charging and communication of charging facility, and effectively normalize the compatibility and communication consistency for interface of charging facility from different operators.
\end{abstract}

\section{Introduction}

In recent years, the carbon dioxide emissions has continued to rise, and global climate change becomes the most significant challenge that the human being faces in present day and a very long time in future, therefore, it becomes the important planning of national development to solve environmental pollution problems and realize energy conservation and emission reduction, so the electric vehicles becomes a new development direction of automobile industry as one effective measure for energy conservation and emission reduction. As of the end of 2015, the sales volume of electric vehicles of our country had accumulated 497,000 units. Meanwhile, our country had built 3,600 charging/swap stations and 50,000 charging piles. In 2015, the General Office of the State Council of the People's Republic of China issued Guiding Suggestions on Speeding up Construction of Charging Infrastructure for Electric Vehicles (GBF (2015) No.73), and four ministries issued Development Guidance for Charging Infrastructure for Electric Vehicles (2015-2020), and put forward that 12,000 charging stations and 4,800,000 charging piles will be increased by 2020, to satisfy charging needs of 5,000,000 electric vehicles.

With the increase in the number and brands of electric vehicles and the large-scale construction of charging infrastructure, a problem arises that the electric vehicles with different brands and the charging piles with various models cannot be normally charged, which has attracted great attention from the auto industry at home and abroad, the power sector and other relevant industries. This is an urgent problem to be solved. On December 27, 2015, the issuance of the five new national standards such as GB/T 18487.1 etc provides a standard basis for the detection of charging interface. The problem of charging interface compatibility after the issuance of new national standard is put forward respectively in [1]-[2] of the References, and the difficulty in unifying charging interfaces is also raised. An introduction to the charging interface standards of electric vehicles is given in [3]-[7] of the References in conjunction with the actual situation of the State Grid Corporation of China and the popularization and application needs of electric vehicles. The detection methods and techniques in [8]-[9] of the References provides a theoretical basis for the detection system. The key technology of AC/DC charging interface detection system is put forward in the thesis in conjunction with the five new national standards and the detection methods and techniques of other fields, solving the problems such as charging interface compatibility etc. 


\section{Standards of Basis for Detection System}

The charging interfaces of electric vehicles are detected mainly according to the newly issued five national standards: GB/T 18487.1-2015 Conductive Charging System of Electric Vehicles. Part 1: General Requirements, GB/T 20234.1-2015 Connecting Devices for Conduction and Charging of Electric Vehicles. Part 1: General Requirements, GB/T 20234.2-2015 Connecting Devices for Conduction and Charging of Electric Vehicles. Part 2: AC Charging Interface, GB/T 20234.3-2015 Connecting Devices for Conduction and Charging of Electric Vehicles. Part 3: DC Charging Interface and GB/T 27930-2015 Communication Protocol between Non-Vehicle-Mounted Conductive Charger and Battery Management System of Electric Vehicles. The conductive charging interface of electric vehicle and communication protocol standard are fundamental criteria to guarantee the interconnection of electric vehicles and charging infrastructure. The new standard further details the functional specifications of charging system and promotes charging safety; further improves the performance index of charging standard and enhances charging compatibility, providing the basic support for the research in the detection methods of AC/DC charging interface consistency of electric vehicles.

\section{Detection Methods of Charging Interface Consistency}

With the continuous development of robot technology, the team at home and abroad with the basic function can play football and in gradually to pick the ball function development. Pick the ball design break through the traditional $2 \mathrm{~d}$ game model, this makes robot can directly hit the opponent ball and don't have to go around, this simplified decision-making, increase attack and breakthrough are quite useful. But because of the limits of the robots, how to make robots in the limited space in both playing and picking the ball is a big test of the structure design.

The detection of AC/DC charging interface consistency of electric vehicles includes the research in the detection method of simulated vehicle end against charging pile interface and the study in the detection precautions of simulated pile tip against the charging interface of electric vehicle. The detection of charging interface consistency mainly includes the tests in the aspects such as physics, electric, communication, function and safety, etc. The physical and electrical performance of charging interface can be verified in such methods as observation, insertion \& extraction, comparison and thermal test etc; the communication interoperability of charging interface is detected and verified mainly based on national standards in interaction and analog methods in the thesis. See Fig. 3-1.

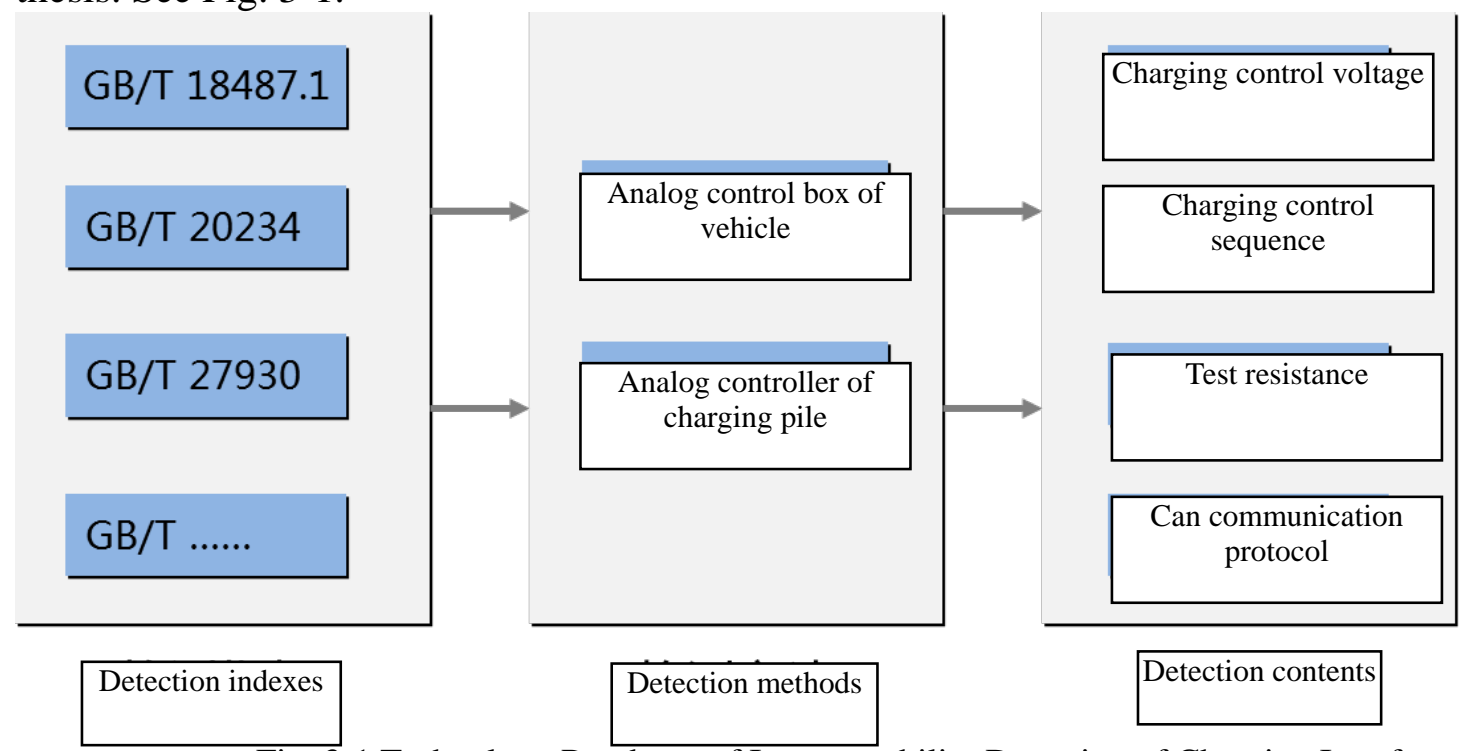

Fig. 3-1 Technology Roadmap of Interoperability Detection of Charging Interface 


\section{Detection Method of Communication Interoperability of DC Charging Interface}

The charging control voltage and sequence of DC charging interface are tested mainly by means of analog control box of vehicle in the thesis. It is judged whether the charging interface of the charging pile has compatibility or not according to the interface communication protocol requirements stipulated in the new national standards.

\section{Detection Conditions and Requirements on DC Charging Interface of Charging Pile}

For the detection of DC charging interface consistency, the analog control box of vehicle is used to test DC charging control voltage and sequence of non-vehicle-mounted chargers. The test conditions shall cover rated condition and failure test beyond tolerance range. The analog control box of vehicle shall meet the requirements as follows:

(1) The test resistance of vehicle control analog circuit shall conform to the requirements in Table 3-1, and the precision of the test resistance shall be no less than $\pm 0.2 \%$. Except the specially explained test items, nominal value is selected for testing resistance in a test;

(2) S+, S- are CAN communication lines, and the communication protocol shall comply with the provisions in GB/T 27930-2015;

(3) The voltage of pull-up voltage $\mathrm{U} 2$ is $12 \mathrm{~V} \pm 0.6 \mathrm{~V}$.

Table 3-1 Test Resistance of Vehicle Control Analog Circuits

\begin{tabular}{|l|l|l|}
\hline States & $\mathrm{R} 4(\Omega)$ & $\mathrm{R} 5(\Omega)$ \\
\hline upper deviation & 1060 & 1060 \\
\hline beyond tolerance & 1035 & 1035 \\
\hline within tolerance & 1025 & 1025 \\
\hline nominal value & 1000 & 1000 \\
\hline within tolerance & 975 & 975 \\
\hline beyond tolerance & 965 & 965 \\
\hline lower deviation & 940 & 940 \\
\hline
\end{tabular}

\section{Detection Conditions and Requirements on DC Charging Interface of Electric Vehicles}

For the detection of the interface $s$ of electric vehicle, non-vehicle-mounted charger simulators are used in the thesis to test DC charging control voltage and sequence of electric vehicles. The test conditions shall include the rated condition and failure test beyond tolerance range. The charger simulator shall satisfy the following requirements:

(1) K3 and K4 are auxiliary power switches, and the power source is $12 \mathrm{~V} \pm 5 \%, 10 \mathrm{~A}$;

(2) The test resistance of non-vehicle-mounted charger simulator shall meet the requirements in Table 3-2, and the precision of the test resistance shall be no less than $\pm 0.2 \%$. Except the specially explained test items, nominal value is selected for testing resistance in a test;

(3) S+, S- are CAN communication lines, and the communication protocol shall comply with the provisions in GB/T 27930-2015;

(4) The rated voltage of pull-up voltage $\mathrm{U} 1$ is $12 \mathrm{~V}$, and the tolerance is $\pm 0.6 \mathrm{~V}$.

Table 3-2 Test Resistance of Control Analog Circuits in Non-Vehicle-Mounted Charger

\begin{tabular}{|l|l|l|l|}
\hline States & $\mathrm{R} 1(\Omega)$ & $\mathrm{R} 2(\Omega)$ & $\mathrm{R} 3(\Omega)$ \\
\hline upper deviation & 1060 & 1060 & 1060 \\
\hline beyond tolerance & 1035 & 1035 & 1035 \\
\hline within tolerance & 1025 & 1025 & 1025 \\
\hline nominal value & 1000 & 1000 & 1000 \\
\hline within tolerance & 975 & 975 & 975 \\
\hline beyond tolerance & 965 & 965 & 965 \\
\hline lower deviation & 940 & 940 & 940 \\
\hline
\end{tabular}




\section{Detection Method of AC Charging Interface}

Vehicle analog control box is used in the thesis to test AC charging control voltage and sequence of AC charging pile. The test conditions shall include the rated condition and failure test beyond tolerance range. The vehicle analog control box shall meet the following requirements:

(1) Table 3-3 shows various states of test control resistance nominal and deviation, and the precision of the test resistance shall be no less than $\pm 0.2 \%$. Except the specially explained test items, nominal value is selected for testing resistance in other tests;

Table 3-3 Test Resistance of Vehicle Control Analog Circuit

\begin{tabular}{|l|l|l|}
\hline States & $\mathrm{R} 2(\Omega)$ & $\mathrm{R} 3(\Omega)$ \\
\hline upper deviation & 1723 & 4610 \\
\hline nominal value & 1300 & 2740 \\
\hline lower deviation & 909 & 1820 \\
\hline
\end{tabular}

(2) Vehicle control analog circuits of analog vehicle charging given in the Test Specifications for the Conduction Charging Interoperability of Electric Vehicles are to be adopted in Fig. 3-2. The specific is as follows:

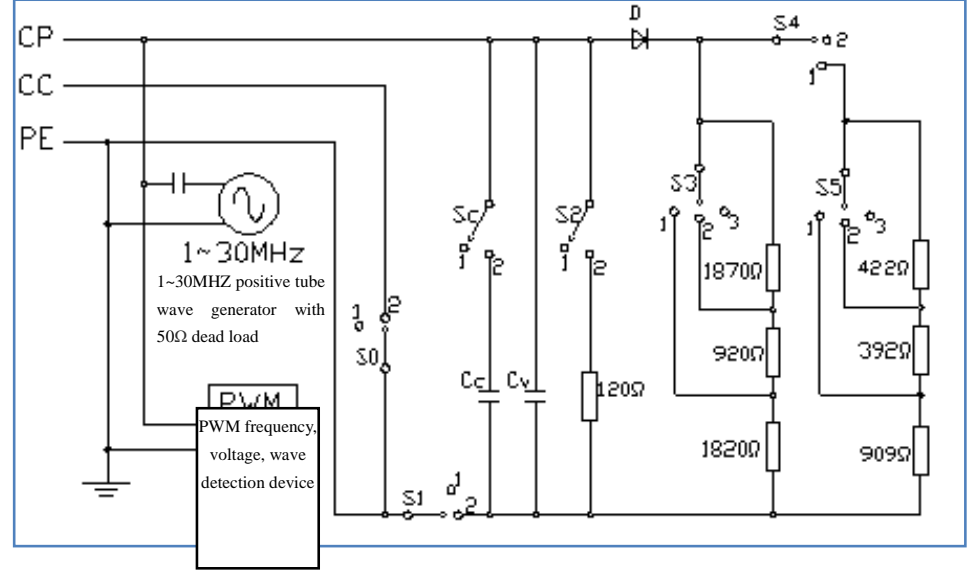

Note 1: test switches adopted shall be low resistance coating terminals;

Note 2: switch S0 is only for the power units of charging mode 3, and adopts A or B connection type; it is opened for simulating CC open circuit protection, and closed for other power units or tests;

Note 3: Sc is used for simulating cable capacitance, and is opened for the power units of C connection type;

Note 4: switch S2 is used for simulating CP grounding test.

Fig. 3-2 Example of Vehicle Control Analog Circuits

(3) A sine-wave generator connects with $50 \Omega$ resistance and $1,000 \mathrm{pF}$ capacitance and has an overlap joint with CP \& PE, and is used for simulating high-frequency signal injection. The output frequency shall cover $1 \mathrm{MHz} 30 \mathrm{MHz}$, the logarithmic stepping width is $4 \%$ and kept for $0.5 \mathrm{~s}$; the output amplitude of sine-wave generator shall be able to be adjusted so as to ensure that the high-frequency voltage peak-to-peak value loaded on CP loop is $2.5 \mathrm{~V}$, and the measuring position is in the power supply socket (for $\mathrm{A}$ and $\mathrm{B}$ connection type) or vehicle plug (for $\mathrm{C}$ connection type);

(4) The maximum value in Table A.5 in GB/T 18487.1-2015 shall be used for Cv and Cc;

(5) The model selection of D shall meet the requirements in Table A.5 in GB/T 18487.1-2015;

(6) The length of the charging cable during a test shall be less than $3 \mathrm{~m}$;

(7) Table 3-4 defines the switch states corresponding to different states during the test of AC charging. It is able to simulate complete charging sequence test and fault test, including test of R2 and R3nominal values and the failure test beyond tolerance range. When guided voltage test is controlled, Switches S1and S4 are used to switch the charging processes State1, State2 and State 3; when nominal values are used to make a test, Switches S3 and S5 are in State 2. 
Table 3-4 Definition of Switch States

\begin{tabular}{|c|c|c|c|c|c|c|c|c|}
\hline $\mathrm{S} / \mathrm{N}$ & $\begin{array}{ll}\text { Charging } & \text { Process } \\
\text { States } & \end{array}$ & Parameter Zone & S0 & S1 & S2 & S3 & S4 & S5 \\
\hline 1 & State 1 & not connected & 1 & 1 & 1 & $\times$ & $\times$ & $x$ \\
\hline 2 & $\begin{array}{l}\text { protective grounding } \\
\text { disconnected }\end{array}$ & $\begin{array}{ll}\text { ground } & \text { wire } \\
\text { disconnected } & \\
\end{array}$ & 1 & 1 & 1 & $x$ & $x$ & $x$ \\
\hline 3 & $\begin{array}{ll}\text { control } & \text { guided } \\
\text { grounding } & \\
\end{array}$ & CP grounding & 2 & 2 & 2 & $x$ & 2 & $x$ \\
\hline 4 & State 2 & \multirow{2}{*}{ nominal value } & 2 & 2 & 1 & 2 & 2 & $x$ \\
\hline 5 & State 3 & & 2 & 2 & 1 & 2 & 1 & 2 \\
\hline 6 & State 2 & \multirow{2}{*}{ upper deviation } & 2 & 2 & 1 & 3 & 2 & $\times$ \\
\hline 7 & State 3 & & 2 & 2 & 1 & 3 & 1 & 3 \\
\hline 8 & State 2 & \multirow{2}{*}{ lower deviation } & 2 & 2 & 1 & 1 & 2 & $x$ \\
\hline 9 & State 3 & & 2 & 2 & 1 & 1 & 1 & 1 \\
\hline
\end{tabular}

\section{Conclusion}

The key technology of charging interface consistency detection system of electric vehicle is put forward in the thesis. The detection system can detect the interfaces of charging equipment manufactured by different operators and production enterprises. Detection is made to control voltage, charging sequence and communication protocol to verify whether the AC/DC charging pile interfaces conform to new national standards. The compatibility, consistency detection of charging interface can be realized through the research on the key technology of the detection system, preventing the charging interface non-conforming to national standards from flowing into the market, resulting in incompatibility problem of charging interfaces.

\section{References}

[1] Whether the New National Standards Implemented are for Charging Interface of Electric Vehicle to be Test [J]. Power World, 2016, 01:4.

[2] Xie Hongchen. The Issued New National Standards for Charging Interface of Electric Vehicles will be Compatible with Charging Pile [J]. Research and Utilization of Energy, 2016, 01:14.

[3] Jia Junguo, Ni Feng. Discussion on the Standardization of Charging Interface of Electric Vehicle [J]. Power System Automation, 2011, 08:76-80.

[4] Gao Yipan. Tracking Study on Charging Interface International Standard of Electric Vehicles under the Framework of Electric Accessory Standard System[J]. Household Electrical Appliances, 2014, 01:12-16.

[5] Zhen Zijian, Meng Xiangfeng. Analytical Investigation on the Technology and Standard Comparison of AC Charging Interface of Domestic and International Electric Vehicles [J]. Automotive Engineering Journal, 2012, 01:1-7.

[6] Liu Yongdong. Interpretation on Unified Electric Vehicle Charging Interface and Communication Protocol Standard-Charging Interface and Protocol Standard[J]. Chinese Power Enterprise Management, 2012, 07:24-25.

[7] Xiao Wei. Comparisons of Chinese-American Electric Vehicle AC Charging Interface and Control Guided Circuits [A]. Chongqing Automotive Engineering Society; Annual Meeting Memoir of 2012 Chongqing Automotive Engineering Society [C]. Chongqing Automotive Engineering Society, 2012:6. 
[8] Li Yan, Bao Ninghai. Research on Key Technology of LTE Network S1/X2 Interface Consistency Test [J]. Modern Telecom Technology, 2011, 09:31-35.

[9] Li Gang, Zhou Yibo, Qing Boyuan. Discussion on Terminal Detection Method of Guangxi Power Demand Side Management [J]. Guangxi Power, 2012, 05:53-56.

Note: the thesis is derived from the science and technology guideline project of the State Grid Corporation of China Key Technology and Verification of AC/DC Charging Interface Consistency Detection of Electric Vehicles. 